\title{
Results of a phase IIa clinical trial of an endogenous anti-inflammatory molecule, chaperonin 10, in multiple sclerosis
}

Broadley SA,${ }^{1,2}$ Vanags D, ${ }^{3}$ Williams B, ${ }^{3}$ Johnson B, ${ }^{3}$ Feeney D, ${ }^{3}$ Griffiths L, Shakib S, ${ }^{4}$ Brown G, ${ }^{4}$ Coulthardt A, ${ }^{5,6}$ Mullins $\mathrm{P},{ }^{7}$ Kneebone $\mathrm{C} .{ }^{4}$

\footnotetext{
${ }^{1}$ School of Medicine, Gold Coast Campus, Griffith University QLD 4222, Australia

${ }^{2}$ Gold Coast Hospital, 108 Nerang Street, Southport QLD 4215, Australia

${ }^{3}$ CBio Ltd, 85 Brandl Street, Eight Miles Plains QLD 4113, Australia

${ }^{4}$ Royal Adelaide Hospital, North Terrace, Adelaide SA 5000, Australia

${ }^{5}$ Royal Brisbane and Women's Hospital, Herston Road, Herston QLD 4006 Australia

${ }^{6}$ University of Queensland, St Lucia QLD 4072, Australia

${ }^{7}$ Department of Statistics, University of Auckland, Private Bag 92019, Auckland 1142, New Zealand
}

\author{
Corresponding author: $\quad$ Associate Professor Simon Broadley \\ School of Medicine, Gold Coast Campus \\ Griffith University QLD 4222 \\ AUSTRALIA \\ Tel.: + $61(0) 755527701$ \\ Fax: + $61(0) 755529197$ \\ Email: $\underline{\text { simon.broadley@griffith.edu.au }}$
}

Running Title: $\quad$ Cpn10 safety in MS

Keywords: Multiple Sclerosis [41]; Clinical trial, Randomized controlled (CONSORT agreement) [20]; Chaperonin 10; Heat Shock Proteins; Treatment

Word Count: 3,171 


\begin{abstract}
Chaperonin 10 is a mitochondrial molecule involved in protein folding. The aim of this study was to determine the safety profile of Cpn10 in patients with MS. Fifty patients with relapse-remitting or secondary progressive MS were intravenously administered $5 \mathrm{mg}$ or 10mg of Cpn10 weekly for 12 weeks in a double-blind, randomized, placebo controlled, phase II trial. Clinical reviews, including Expanded Disability Status Scale and MRI with Gadolinium were undertaken every 4 weeks.

Stimulation of patient peripheral blood mononuclear cells with lipopolysaccharide ex vivo was used to measure the in vivo activity of Cpn10. No significant differences in the frequency of adverse events were seen between treatment and placebo arms. Leukocytes from both groups of Cpn10-treated patients produced significantly lower levels of critical proinflammatory cytokines. A trend towards improvement in new Gadolinium enhancing lesions on MRI was observed, but this difference was not statistically significant. No differences in clinical outcome measures were seen. Thus, Cpn10 is safe and well tolerated when administered to MS patients for 3 months however a further extended phase II study primarily focused on efficacy is warranted.
\end{abstract}




\section{Introduction}

Multiple sclerosis is a chronic, inflammatory condition of the central nervous system, which affects approximately 2.5 million people worldwide[1]. It is frequently debilitating and definitive treatment for the condition remains elusive. Whilst much remains unknown regarding the underlying aetiology of multiple sclerosis, most authors agree that a disturbance of the immune system and an autoimmune response directed at components of the central nervous system are crucial aspects of the pathogenesis[1]. Pathological studies have identified the oligodendrocyte as a possible early target of a disordered inflammatory response[2]. Careful histological and in vitro experiments have highlighted a role for TNF- $\alpha$ and $\gamma$-interferon with many other cytokines also implicated.

A number of partially successful treatments for multiple sclerosis have been developed. These treatments include T-cell suppressants such as azathioprine[3], mitozantrone[4], glatiramer acetate[5] and $\beta$-interferons[6]; methylprednisolone, a general immunosuppressant[7] and natalizumab, a monoclonal antibody against VLA-4[8]. While many of these pharmacological treatments have been shown to be partially successful in treating multiple sclerosis, routes of administration and adverse effects limit their use. Thus, the search for more effective and safe treatment options for multiple sclerosis continues.

Recently, recombinant chaperonin 10 (Cpn10), has been shown to suppress the inflammatory response and improve clinical outcome measures in experimental autoimmune encephalomyelitis, a mouse model of multiple sclerosis $[9,10]$. Cpn10 was originally identified as early pregnancy factor in the serum of pregnant mammals, including humans[11]. Intracellularly, Cpn10 interacts with Cpn60 (Hsp60) to promote protein folding in mitochondria. Extracellular Hsp60 has been shown to act as an agonist for a group of pro-inflammatory innate immune receptors known as toll-like receptors (TLRs)[12, 13]. In contrast, Cpn10 may elicit its anti-inflammatory activity by blocking the interaction between Hsp60 and TLRs[14]. Thus, it has been postulated that Cpn10 may have a systemic immunomodulatory role similar to its role in the down-regulation of the immune system observed in pregnancy. Additional studies have indicated that chaperonin 10 suppresses the 
expression of adhesion molecules[15] and appears to affect trafficking of lymphocytes in the central nervous system[16]. More recently, Cpn10 has been demonstrated to promote survival of prooligodendrocytes from neonatal rat brain[17].

In early phase I studies, administration of Cpn10 demonstrated no significant adverse events in subjects. Similarly, the drug was well tolerated when administered intravenously in single and multiple doses over a period of up to five days at doses ranging from $1 \mathrm{mg}$ to $10 \mathrm{mg}$ in both healthy volunteers and patients with multiple sclerosis[18]. Pharmacological studies indicated that Cpn10 had a half-life of approximately 1 hour, when administered intravenously, but was active for up to 4 days in reducing levels of TNF- $\alpha$ in lipopolysaccharide activated peripheral blood mononuclear cell (PBMC) assays[18]. The aim of the present study was to explore the adverse event profile and tolerability of Cpn10 in a cohort of patients with multiple sclerosis over a longer period of time. In addition, pharmacodynamic and clinical responses were monitored using a variety of measures.

\section{Patients and Methods}

\section{Subjects}

Subjects were patients with relapse remitting or secondary progressive multiple sclerosis meeting the McDonald diagnostic criteria[19] but also meeting the additional criterion of having abnormalities on MRI brain as defined by Paty[20]. Subjects were required to be aged 18 to 60 years at enrolment and have an Expanded Disability Status Scale (EDSS)[21] less than 7.0. All subjects gave written informed consent.

Subjects were excluded if another definable cause for their presentation emerged, they had primary progressive multiple sclerosis or an isolated clinical syndrome, they had inadequate venous access, they were not able to give informed consent, they had a contraindication to MRI scanning or were intolerant of MRI scanning, they were unable to receive Gadolinium, or they had another illness or past history of illness which might have interfered with assessments or the subjects ability to complete the study. Additional exclusion criteria were a normal MRI brain at screening, exacerbation of 
multiple sclerosis during the four week screening period, receipt of any disease modifying therapy during the previous 3 months, receipt of any experimental therapy within the previous 30 days, a positive pregnancy test, significantly abnormal hematological or biochemical parameters, an antinuclear antibody titre of 1:80 or greater, inability or unwillingness to take adequate contraceptive precautions, or donation or loss of more than $500 \mathrm{ml}$ of blood in the previous 12 weeks.

Subjects were enrolled at two centres, CMAX, Royal Adelaide Hospital and Griffith University, Gold Coast, Australia. Institutional human research ethics committee approval was obtained at both sites. The study was registered with the Australian Clinical Trials Registry (ACTRN0 12606000037505).

\section{Study Design}

This was a parallel, dose comparison, randomized, double-blind, placebo-controlled trial of Cpn10. The two treatment arms received either Cpn10 twice weekly or once weekly alternating with placebo. The control arm received placebo twice weekly. The active treatment was $5 \mathrm{mg}$ of recombinant Cpn10 in $50 \mathrm{mM}$ Tris- $\mathrm{HCl}$ buffer with $150 \mathrm{mM} \mathrm{NaCl}$ at $\mathrm{pH} 7.6$ diluted to $10 \mathrm{ml}$ in sterile water for injection and administered as a slow injection intravenously over 2 minutes. The placebo consisted of the buffering solution alone diluted in sterile water for injections. Both solutions were clear and colorless. Active product prepared by BresaGen Ltd (Adelaide, Australia) and placebo were diluted into vials by Formatech Inc (Boston, USA). The randomization schedule was prepared on a clustered design based on each site by CMAX (Adelaide, Australia). Eligible subjects were assigned a treatment ID at enrolment into the study and treatment codes were held in sealed envelopes at each site. Thus the study was blinded to both the subject and study investigators.

The Cpn10 dose of $5 \mathrm{mg}$ was chosen based on phase I data which indicated that this dose produced the greatest decrease in lipopolysaccharide induced production of TNF- $\alpha$ without any undue adverse effects[18].

Subjects were seen for initial screening 4 weeks prior to enrolment into the treatment phase of the study. Details of full medical history and multiple sclerosis history were taken including details of 
relevant prior investigations. A physical examination including EDSS[21] and an ECG were undertaken. Samples for blood count, biochemical profile, thyroid function tests, thyroid antibodies, anti-nuclear antibodies, HIV, hepatitis B and C, drug and alcohol screen, urinalysis and pregnancy testing were taken. A screening MRI of brain was also undertaken. These tests together with baseline MSIS-29[22] and pharmacokinetic, pharmacodynamic and anti-Cpn10 antibody samples were repeated prior to enrolment into the treatment phase (week 0). Pharmacokinetic measurements were taken at 30 minutes following dosing on weeks 1, 4, 8 and 12. Serum Cpn10 levels were determined using an in-house validated sandwich ELISA method, with a detection limit of $0.195 \mathrm{ng} / \mathrm{ml}$. Blood was taken for pharmacodynamic measurement on weeks 1 and 12 at 8 hours post $\mathrm{Cpn} 10$ administration, and on weeks 4 and 8 as trough samples. For the pharmacodynamic response, an in vitro assay of cytokine generation by lipopolysaccharide stimulated PBMC was used as a measure of the in vivo biological activity of Cpn10. PBMC were isolated and stimulated, and secreted cytokine levels were quantitated by Cytometric Bead Array technology (Human Inflammation kit, BD CBA software, BD Biosciences) as previously described[14, 23]. Testing for anti-Cpn10 antibodies was performed using a validated, titre-based ELISA method developed in-house. Serial dilutions of serum samples were added to microtitre plates coated with Cpn10. Cpn10-specific patient antibodies that bound to the plates were detected with an anti-human Ig antibody (GE Healthcare). A four-fold increase in antibody titre above baseline samples was taken as significant. This level of significant titre rise was determined in two ways. Empirically from experience with the assay which had demonstrated a random variance in repeat testing of samples of up to two-fold and from previously published reports of similar titre-based assays indicating that a four-fold increase is an appropriate cut-off[24].

Treatment/placebo was administered twice weekly for 12 weeks. Vital signs were monitored before and after each treatment administration. Neurological assessment with EDSS, blood tests, MRI and review of relapses and adverse events was undertaken every 4 weeks during the treatment phase and at four weeks after discontinuation of treatment. The MSIS-29 was also repeated at the end of the treatment phase (week 12). The protocol schedule is summarized in Figure 1. 


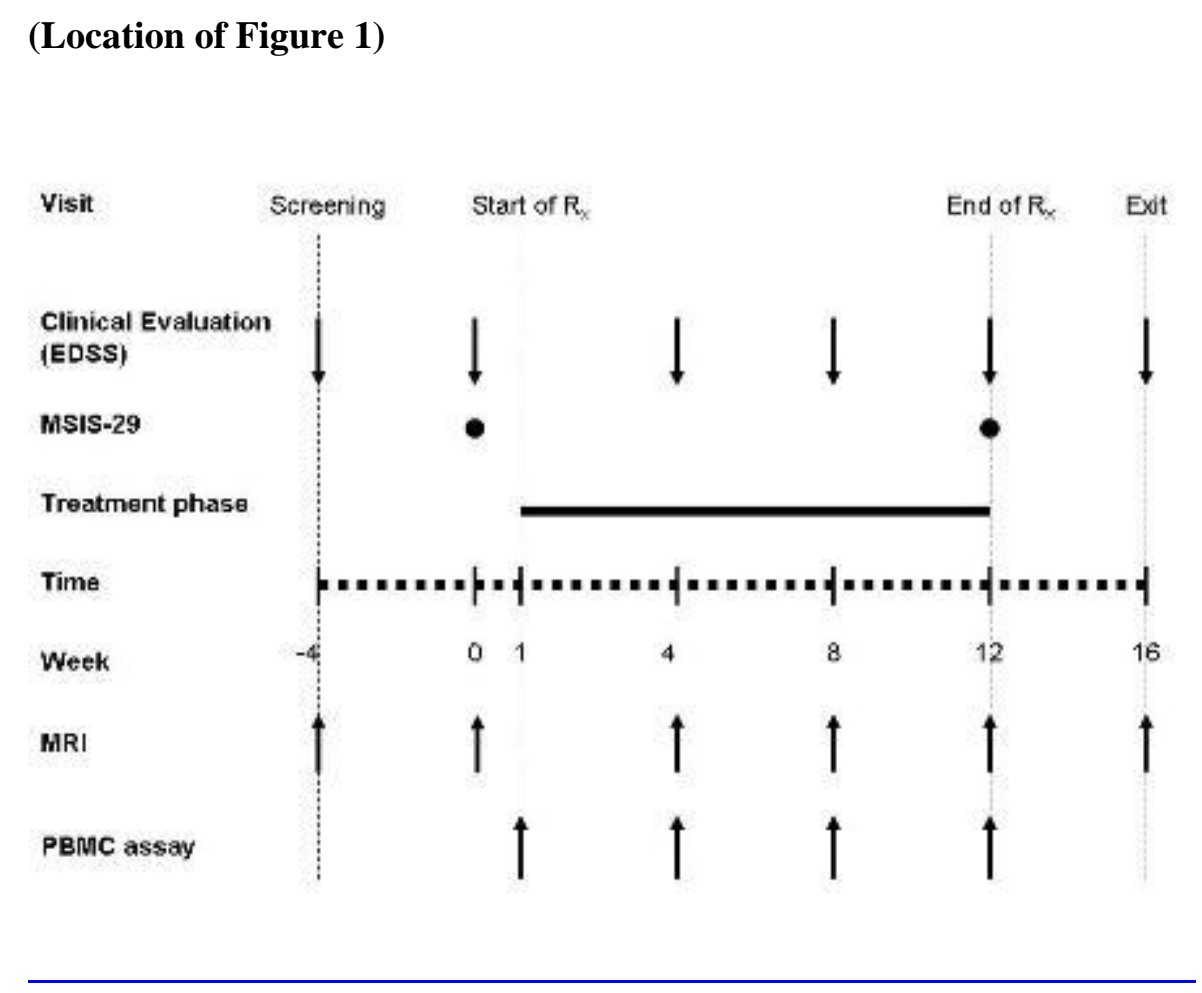

Subjects were encouraged to contact the study coordinators if any significant new symptoms occurred and an unscheduled visit was arranged to record any relapse or significant adverse event. Other adverse events were recorded by the study staff at each visit or drug administration session and then reviewed by the principal investigator at each site.

The primary endpoint for the study was the adverse event profile. Secondary endpoints were the pharmacodynamic response, number and volume of new Gadolinium enhancing lesions on MRI during study compared to baseline, relapse rate, EDSS and MSIS-29.

\section{Imaging}

MRI scans were performed using a predefined protocol and included T1 weighted axial scans with and without Gadolinium, proton density axial, T2 weighted axial, T2 weighted sagittal and FLAIR sequence axial images. Scans were performed on either a Siemens Magnetom Vision 1.5T or GE Signa Genesis $1.5 \mathrm{~T}$ scanner. All imaging data was reviewed by a single reviewer using semiautomated region of interest software (OsiriX). For each time point the following data were collected; total number of Gadolinium enhancing lesions, number of new Gadolinium enhancing lesions, number of previously Gadolinium enhancing lesions and total area of Gadolinium enhancing lesions. 
In view of the short duration of the study, no interim statistical analysis was planned. A decision was made to continue the study unless a serious adverse event occurred and it was felt, on review by the principal investigator in consultation with the relevant ethics committee, that this was likely to be related to the administration of drug.

\section{Statistical analysis}

Power calculations indicated that a total of 50 subjects with $40 \%$ in each treatment arm and $20 \%$ in the placebo arm would have $80 \%$ power to show a pharmacodynamic response, $50 \%$ power to show a change in new enhancing lesions on MRI and $30 \%$ power to detect a clinical change in relapse rate assuming an $\alpha$ of 0.05 with $20 \%$ change in the variable measured, or 1.0 change in the case of the EDSS, as being clinically significant.

All statistical analyses were performed on the intention to treat population using all available safety and efficacy data. Safety data were analyzed on the basis of treatment emergent adverse events. Baseline characteristics were defined at the pre-treatment screening visit (Week 0). Data were analyzed for variance and parametric analysis of variance performed, with respect to patients and doses of Cpn10, where homogeneity was found. Where homogeneity was not found non-parametric analysis of variance was performed, to compare any differences between treatment groups. The null hypothesis stated that there was no effect of Cpn10 on disease outcome variables and a p-value of $<0.05$ was taken as significant. No correction for multiple testing was made and therefore significant results can only be taken as indicative and not definitive. Because of the small numbers of patients and the high variability in counts of new Gadolinium enhancing lesions, this data has been reported in comparison to the activity seen at baseline allowing each subject to act as their own control. Two analyses were performed on the MRI data, one for the incidence of new lesions and the other for the volume of the total number of lesions. The incidence of new enhancing lesions and total number of lesions were discrete response variables and the distribution of the number of such lesions occurring at any time point was reasonably modeled as a negative binomial variate. 
For the pharmacodynamic data, at each time point following screening (Week 1, 4, 8 and 12) an ANOVA was conducted comparing the change in parameter value from baseline across the three treatment groups. In addition, at each time point for each treatment group, a paired test (one-sided) gave an indicative comparison of the parameter value with the baseline (screening) value.

\section{Results}

The numbers of patients screened and enrolled, and their randomized allocation is summarized in Figure 2. Reasons for exclusion are listed in Figure 2. Following randomization, 20 subjects received $5 \mathrm{mg}$ of Cpn10 twice weekly, 19 subjects received Cpn10 (5 mg) alternating with placebo each week and 11 subjects received placebo twice weekly. There were no significant differences in baseline characteristics, however, patients in the placebo group were generally older, were more likely to be male and had less active disease (Table 1).

\section{(Location of Figure 2 and Table 1)}

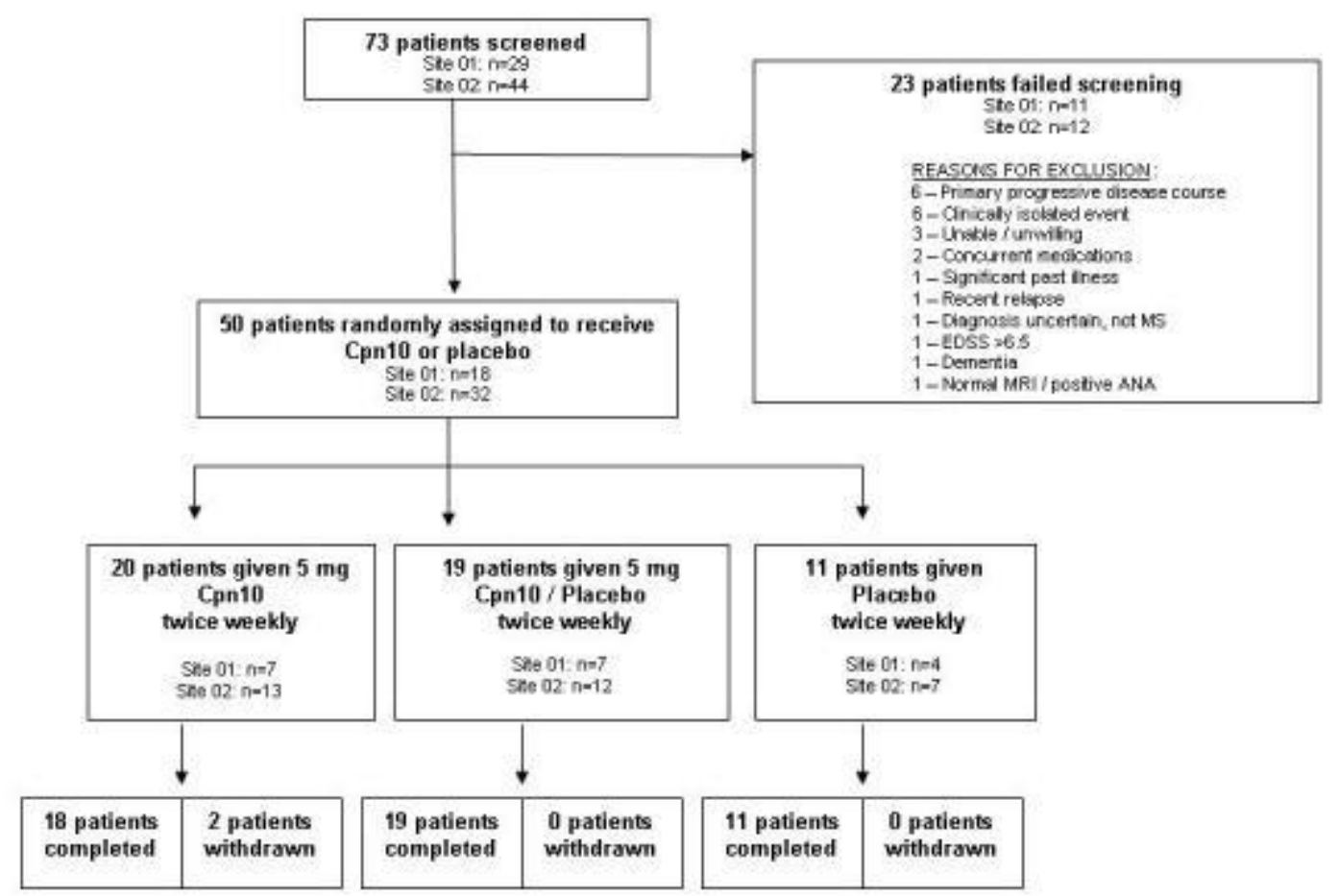


$\underline{\text { Tables }}$

Table 1. Baseline characteristics.

\begin{tabular}{lcccc}
\hline \multicolumn{1}{c}{ Characteristic } & $\underline{\text { Cpn10 }}$ & $\underline{\text { Cpn10/Pla }}$ & $\underline{\text { Placebo }}$ & $\underline{\text { Total }}$ \\
\hline$\underline{N}$ & $\underline{20}$ & $\underline{19}$ & $\underline{11}$ & $\underline{50}$ \\
$\underline{\text { Age- median (range) }}$ & $\underline{4.5(31-60)}$ & $\underline{44.0(25-57)}$ & $\underline{49.0(34-58)}$ & $\underline{46.0(25-60)}$ \\
$\underline{\text { Female-no. (\%) }}$ & $\underline{15(75)}$ & $\underline{15(79)}$ & $\underline{7(64)}$ & $\underline{37(74)}$ \\
$\underline{\text { Dis. dur. }{ }^{a}>10 \text { yrs - no. (\%) }}$ & $\underline{11(55)}$ & $\underline{11(58)}$ & $\underline{6(55)}$ & $\underline{28(56)}$ \\
$\underline{\text { EDSS }{ }^{b}-\text { median (range) }}$ & $\underline{3.5(0.0-6.5)}$ & $\underline{4.0(0.0-6.0)}$ & $\underline{3.5(2.0-6.5)}$ & $\underline{3.5(0.0-6.5)}$ \\
$\underline{\text { Total relapses }>3-\text { no. (\%) }}$ & $\underline{10(50)}$ & $\underline{9(47)}$ & $\underline{4(38)}$ & $\underline{23(46)}$ \\
$\underline{\text { Relapse remitting-no. (\%) }}$ & $\underline{15(75)}$ & $\underline{15(79)}$ & $\underline{6(55)}$ & $\underline{36(72)}$ \\
$\underline{\text { Secondary prog. }{ }^{c}-\text { no. (\%) }}$ & $\underline{5(25)}$ & $\underline{4(21)}$ & $\underline{5(45)}$ & $\underline{14(28)}$ \\
$\underline{\text { New enhancing MRI lesions }}{ }^{d}$ & $\underline{0.4}$ & $\underline{1.0}$ & $\underline{0.18}$ & $\underline{0.58}$ \\
\hline
\end{tabular}

$\stackrel{ }{a}$ Disease duration

${ }^{b}$ Expanded Disability Status Scale

${ }^{c}$ Secondary progressive

${ }^{d}$ Mean number of new Gadolinium enhancing lesions on MRI during screening

There were no significant differences in the frequency of adverse events between the three treatment arms (Table 2). The frequencies of specific symptoms, where the overall frequency was $10 \%$ or more, are shown in Table 2 for each treatment arm. There were no significant abnormalities detected on laboratory testing and no clear pattern was seen between the three treatment arms in the 14 minor abnormalities which were detected (data not shown).

(Location of Table 2)

Table 2. Treatment emergent adverse events by system and individual symptoms. 


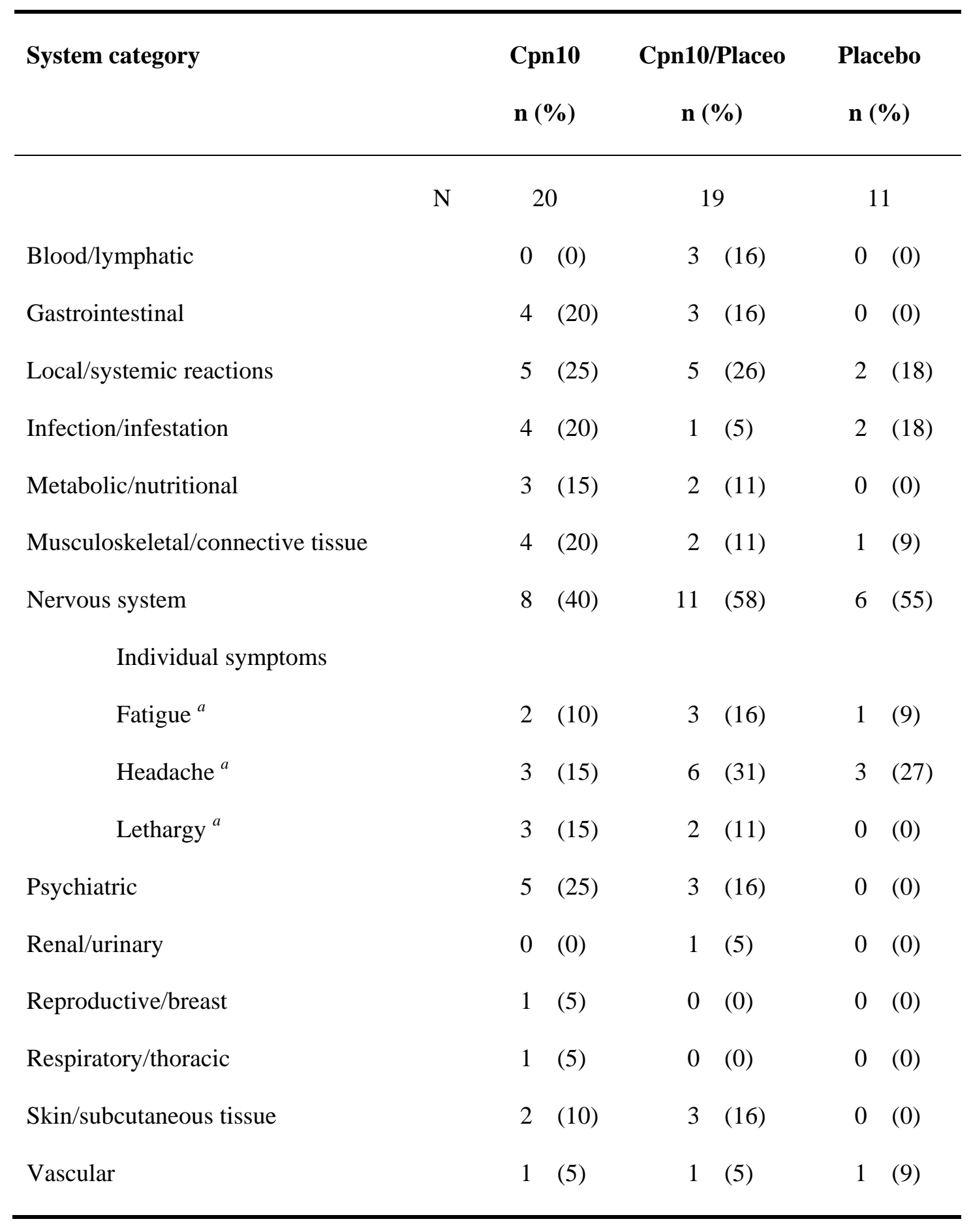

${ }^{a}$ Treatment emergent events for individual symptoms where overall frequency was $10 \%$ or more.

There were two patient withdrawals; both were on active treatment twice per week. In one patient, gastrointestinal symptoms, which may have been related to the administration of drug, was the cause for withdrawal. In the second, dyspnoea without an identifiable underlying cause was the reason. 
Due to logistical and technical problems associated with the isolation of viable PBMC, the sample size for pharmacodynamic measurements was reduced in the placebo $(\mathrm{n}=5)$ and both $\mathrm{Cpn} 10$ treatment cohorts $(\mathrm{n}=7$ each). PBMC isolated from patient blood samples were stimulated with LPS and levels of secreted TNF- $\alpha$, IL-1 $\beta$, IL-6, IL-8, IL-10 and IL-12 were measured. Levels of TNF- $\alpha$ and IL-1 $\beta$ were significantly lower in the Cpn10/Placebo cohort by week 8 of treatment compared to predose (Figure 3). While levels of TNF- $\alpha$ did not remain significantly inhibited, levels of IL-1 $\beta$ remained significantly lower in this cohort at week 12 . Similarly, by week 8 , IL-8 and IL-10 levels were significantly lower in the Cpn10 cohort nevertheless, levels of these cytokines returned to baseline by week 12. Changes in levels of IL-6 and IL-12 were not observed in Cpn10-treated cohorts (data not shown).

\section{(Location of Figure 3)}
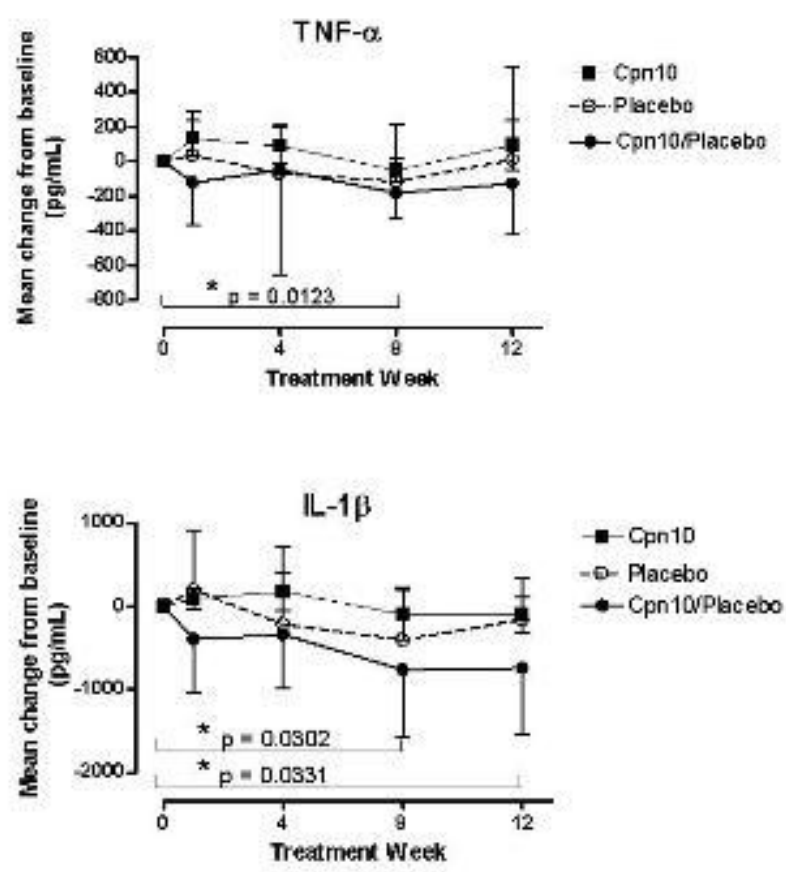

At exit evaluation (Week 16), 3 of 39 (8\%) subjects on treatment (1 on Cpn10, 2 on Cpn10/Placebo) had a four-fold or greater rise in antibody titre. No increase in antibody of this magnitude was seen in the placebo group, although a three fold increase in titre was observed in one patient receiving placebo.

The cumulative change in mean numbers of new Gadolinium enhancing lesions compared to baseline for each group are shown in Figure 4. A trend towards a reducing number of lesions in the two active 
treatment arms and a rise in lesion number in the placebo arm were detected. The lesion volume data did not show this trend (data not shown). One patient in the Cpn10/Placebo arm had a significant relapse which began on day 1 and developed 11 new-enhancing lesions at Week 4, and was therefore considered an outlier from the other patients in this group (Figure 4). It is unlikely that this was a treatment effect, although the possibility cannot be discounted.

\section{(Location of Figure 4)}

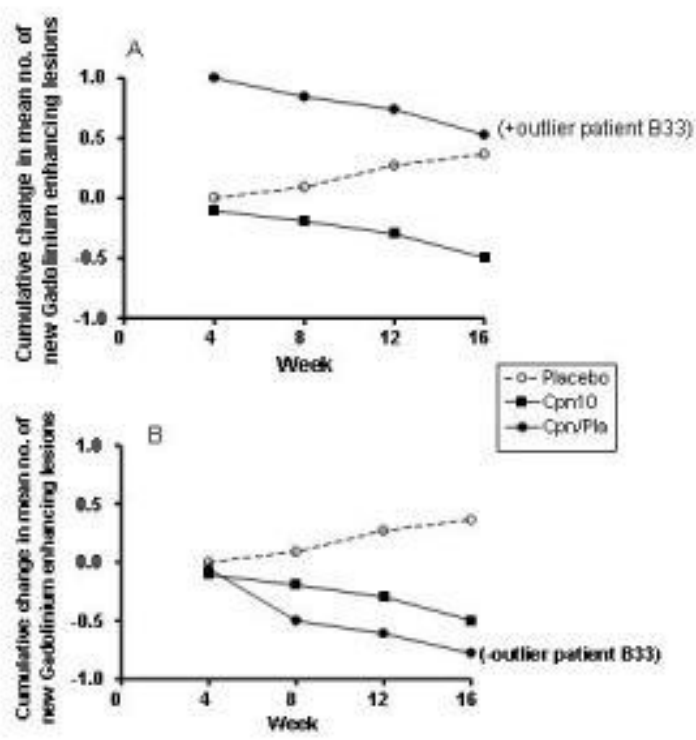

Relapses occurred in 6 of $50(12 \%)$ subjects with 0 of $20(0 \%)$ in the twice weekly Cpn10 treatment group, 5 of $19(26 \%)$ in the Cpn10/Placebo treatment group and 1 of $11(9 \%)$ in the Placebo treatment group. These differences were not statistically significant, however it should be noted that in 3 of the 5 subjects suffering a relapse in the Cpn10/Placebo arm, the relapse occurred within the first week of treatment. There were no significant changes in EDSS or MSIS-29 from pre-study (Week 0) to post study (Week 12) assessments (Table 3).

\section{(Location of Table 3)}

Table 3. EDSS and MSIS-29 pre- and post-treatment. 


\begin{tabular}{|c|c|c|c|c|c|c|c|}
\hline \multirow[t]{2}{*}{ Measurement } & \multirow[t]{2}{*}{ Week } & \multicolumn{2}{|c|}{ Cpn10 } & \multicolumn{2}{|c|}{ Cpn10/Placebo } & \multicolumn{2}{|c|}{ Placebo } \\
\hline & & Mean & (SD) & Mean & (SD) & Mean & (SD) \\
\hline & $\mathrm{N}$ & & $0^{a}$ & & 19 & & 1 \\
\hline \multirow[t]{2}{*}{ EDSS } & 0 & 3.8 & $(2.0)$ & 3.2 & (1.8) & 3.8 & (1.6) \\
\hline & 12 & 3.8 & $(2.2)$ & 3.3 & (1.6) & 3.8 & (1.6) \\
\hline \multirow[t]{2}{*}{ MSIS-29 } & 0 & 61.1 & $(15.8)$ & 53.2 & (13.6) & 64.0 & (25.6) \\
\hline & 12 & 59.5 & (19.7) & 53.0 & (23.1) & 60.5 & (29.4) \\
\hline
\end{tabular}

${ }^{a}$ One subject not assessed at Week $12(\mathrm{n}=19)$.

\section{Discussion}

In this study, intravenous administration of Cpn10 at two dosing regimens, twice weekly and once weekly, over a 12 week period proved to be safe and well tolerated. There were no significant differences in the frequency of adverse events between the three treatment arms. In addition, there was no indication of any deterioration on secondary clinical outcome measures in the active treatment arms.

We did not observe any significant changes in either MRI or clinical markers of disease activity over the 12 weeks of the treatment phase. There was a trend towards fewer new Gadolinium enhancing lesions in the two active treatment arms compared to a minor increase in the Placebo group when lesion counts were normalized for pre-treatment MRI activity, however, this change was not significant.

A recent study of Cpn10 in rheumatoid arthritis has shown promising results with improvements in clinical measures of disease activity being seen in 6 of 7 (86\%) subjects receiving the highest dose of treatment[23]. In the rheumatoid arthritis trial, higher doses of Cpn10 ranging from $5 \mathrm{mg}$ twice weekly up to $10 \mathrm{mg}$ twice weekly were used. Notably clearer degrees of improvement in clinical 
outcomes were observed in the highest dose group. In the current study, attenuated levels of both TNF- $\alpha$ and IL-1 $\beta$ were observed in the Cpn10/Placebo treated arm only. No consistent trends in cytokine profiles were observed across the three treatment arms but this pattern of cytokine response seen in one of the treated arms is consistent with previous phase I[18] and early phase II[23, 25] trials. This suggests that Cpn10 is biologically active in humans at the dosages used in the current study; nevertheless the dosages may not be therapeutically beneficial.

The present study had very low power to detect any clinical effect of treatment and only modest power to detect any change in MRI activity. The short duration of this trial (12 weeks) was necessary because of the previously limited experience with Cpn10 in human subjects. It is well recognised that the clinical efficacy of existing immunomodulatory treatments for multiple sclerosis can take between 3 and 6 months to become apparent and generally lags 3 months behind any change in MRI activity[26]. The inclusion of subjects with secondary progressive disease, even though most were still experiencing relapses, increased the variability of baseline characteristics in this small cohort. The placebo group by chance were older, more likely to be male and had less active disease. Thus, there are a number of reasons why no significant MRI or clinical efficacy was seen, but low numbers, short duration of treatment, variability of study cohort and possibly inadequate dosing, given the data from the rheumatoid arthritis study, are all possible explanations.

In conclusion, we present evidence that $\mathrm{Cpn} 10$ administered intravenously at doses up to $5 \mathrm{mg}$ twice weekly for 12 weeks to subjects with relapsing remitting or secondary progressive multiple sclerosis is safe and well tolerated. We have not found any significant difference in any of the secondary clinical outcome measures. A further phase IIb clinical trial of Cpn10 involving 100-200 subjects over a 12 month period using the higher dose of $10 \mathrm{mg}$ twice weekly is clearly needed in order to assess any therapeutic efficacy for this potential therapy for multiple sclerosis. If the trend towards a reduction in new Gadolinium enhancing lesions seen in the present study were to be confirmed, the prospects for clinical efficacy in multiple sclerosis would certainly be bolstered. 


\section{Acknowledgements}

We would like to thank Julissa Weiss and Dr Jerome Jayasekera for assistance with preparation of the manuscript. This trial was sponsored by CBio Ltd (Brisbane, Australia) and supported in part by a Pharmaceutical Partnerships Program (P3) Grant from the Australian Federal Government.

\section{Disclosure}

BJ, DV, BW and DF are employees of the sponsoring company CBio Ltd (Brisbane, Australia). 


\section{Figure Legends}

\section{Figure 1. Protocol Schedule.}

Timeline showing major clinical and investigational elements of study.

$\mathrm{R}_{\mathrm{x}}=$ treatment

EDSS $=$ Expanded Disability Status Scale

MRI = magnetic resonance imaging

$\mathrm{PMBC}=$ peripheral mononuclear blood cell

\section{Figure 2. Subject Allocation Summary.}

Summary of screening outcome, treatment allocation and study completion in 96 assessed subjects.

\section{Figure 3. Cytokine secretion by patient PBMCs.}

Changes in levels of TNF- $\alpha$ (A) and IL-1 $\beta$ (B) secreted by lipopolysaccharide-stimulated patient PBMCs were determined from levels measured in samples taken at the beginning of the study. Data are mean \pm SD. Means were compared using $t$ test, one-tailed $(* p<0.05)$.

\section{Figure 4. MRI Activity.}

Cumulative change in mean number of new Gadolinium enhancing lesions at 4, 8 and 12 weeks of treatment and at exit evaluation (16 weeks) compared with baseline in three study arms. The data are shown as mean and the outlier was determined statistically using the extreme studentised deviate method for assessing outliers . 


\section{References}

1. Compston A, Coles A Multiple sclerosis. Lancet 2002; 359:1221-1231.

2. Barnett MH, Prineas JW Relapsing and remitting multiple sclerosis: pathology of the newly forming lesion. Ann Neurol 2004; 55:458-468.

3. Massacesi L, Parigi A, Barilaro A, Repice AM, Pellicano G, Konze A, et al. Efficacy of azathioprine on multiple sclerosis new brain lesions evaluated using magnetic resonance imaging. Arch Neurol 2005; 62:1843-1847.

4. Hartung HP, Gonsette R, Konig N, Kwiecinski H, Guseo A, Morrissey SP, et al. Mitoxantrone in progressive multiple sclerosis: a placebo-controlled, double-blind, randomised, multicentre trial. Lancet 2002; 360:2018-2025.

5. Johnson KP, Brooks BR, Cohen JA, Ford CC, Goldstein J, Lisak RP, et al. Copolymer 1 reduces relapse rate and improves disability in relapsing-remitting multiple sclerosis: results of a phase III multicenter, double-blind placebo-controlled trial. The Copolymer 1 Multiple Sclerosis Study Group. Neurology 1995; 45:12681276.

6. Jacobs LD, Cookfair DL, Rudick RA, Herndon RM, Richert JR, Salazar AM, et al. Intramuscular interferon beta-1a for disease progression in relapsing multiple sclerosis. The Multiple Sclerosis Collaborative Research Group (MSCRG). Ann Neurol 1996; 39:285-294.

7. Beck RW, Cleary PA, Anderson MM, Jr., Keltner JL, Shults WT, Kaufman DI, et al. A randomized, controlled trial of corticosteroids in the treatment of acute optic neuritis. The Optic Neuritis Study Group. N Engl J Med 1992; 326:581-588.

8. Polman CH, O'Connor PW, Havrdova E, Hutchinson M, Kappos L, Miller DH, et al. A randomized, placebo-controlled trial of natalizumab for relapsing multiple sclerosis. N Engl J Med 2006; 354:899-910. 
9. Harness J, Cavanagh A, Morton H, McCombe P A protective effect of early pregnancy factor on experimental autoimmune encephalomyelitis induced in Lewis rats by inoculation with myelin basic protein. J Neurol Sci 2003; 216:33-41.

10. Zhang B, Harness J, Somodevilla-Torres MJ, Hillyard NC, Mould AW, Alewood D, et al. Early pregnancy factor suppresses experimental autoimmune encephalomyelitis induced in Lewis rats with myelin basic protein and in SJL/J mice with myelin proteolipid protein peptide 139-151. J Neurol Sci 2000; 182:5-15.

11. Mehta AR, Eessalu TE, Aggarwal BB Purification and characterization of early pregnancy factor from human pregnancy sera. J Biol Chem 1989; 264:2266-2271.

12. Ohashi K, Burkart V, Flohe S, Kolb H Cutting edge: heat shock protein 60 is a putative endogenous ligand of the toll-like receptor-4 complex. J Immunol 2000; 164:558-561.

13. Vabulas RM, Ahmad-Nejad P, da Costa C, Miethke T, Kirschning CJ, Hacker H, et al. Endocytosed HSP60s use toll-like receptor 2 (TLR2) and TLR4 to activate the toll/interleukin-1 receptor signaling pathway in innate immune cells. J Biol Chem 2001; 276:31332-31339.

14. Johnson BJ, Le TT, Dobbin CA, Banovic T, Howard CB, Flores Fde M, et al. Heat shock protein 10 inhibits lipopolysaccharide-induced inflammatory mediator production. J Biol Chem 2005; 280:4037-4047.

15. Zhang B, Walsh MD, Nguyen KB, Hillyard NC, Cavanagh AC, McCombe PA, et al. Early pregnancy factor treatment suppresses the inflammatory response and adhesion molecule expression in the spinal cord of SJL/J mice with experimental autoimmune encephalomyelitis and the delayed-type hypersensitivity reaction to trinitrochlorobenzene in normal BALB/c mice. J Neurol Sci 2003; 212:37-46.

16. Athanasas-Platsis S, Zhang B, Hillyard NC, Cavanagh AC, Csurhes PA, Morton $\mathbf{H}$, et al. Early pregnancy factor suppresses the infiltration of lymphocytes and 
macrophages in the spinal cord of rats during experimental autoimmune encephalomyelitis but has no effect on apoptosis. J Neurol Sci 2003; 214:27-36.

17. McCombe PA Recombinant EPF/chaperonin 10 promotes the survival of O4-positive pro-oligodendrocytes prepared from neonatal rat brain. Cell Stress Chaperones 2008;

18. Broadley SA, Johnson B, Feeney D, Shakib S Phase 1a and 1b clinical trials of chaperonin 10 in healthy volunteers and patients with multiple sclerosis. J Neurol Sci 2005; 238:S223.

19. McDonald WI, Compston A, Edan G, Goodkin D, Hartung HP, Lublin FD, et al. Recommended diagnostic criteria for multiple sclerosis: guidelines from the International Panel on the diagnosis of multiple sclerosis. Ann Neurol 2001; 50:121127.

20. Paty DW, Oger JJ, Kastrukoff LF, Hashimoto SA, Hooge JP, Eisen AA, et al. MRI in the diagnosis of MS: a prospective study with comparison of clinical evaluation, evoked potentials, oligoclonal banding, and CT. Neurology 1988; 38:180185.

21. Kurtzke JF Rating neurologic impairment in multiple sclerosis: an expanded disability status scale (EDSS). Neurology 1983; 33:1444-1452.

22. Hobart J, Lamping D, Fitzpatrick R, Riazi A, Thompson A The Multiple Sclerosis Impact Scale (MSIS-29): a new patient-based outcome measure. Brain 2001; 124:962-973.

23. Vanags D, Williams B, Johnson B, Hall S, Nash P, Taylor A, et al. Therapeutic efficacy and safety of chaperonin 10 in patients with rheumatoid arthritis: a doubleblind randomised trial. Lancet 2006; 368:855-863. 
24. Tasker SA, Treanor JJ, Paxton WB, Wallace MR Efficacy of influenza vaccination in HIV-infected persons. A randomized, double-blind, placebo-controlled trial. Ann Intern Med 1999; 131:430-433.

25. Williams B, Vanags D, Hall S, McCormack C, Foley P, Weiss J, et al. Efficacy and safety of chaperonin 10 in patients with moderate to severe plaque psoriasis: evidence of utility beyond a single indication. Arch Dermatol 2008; 144:683-685.

26. PRISMS Randomised double-blind placebo-controlled study of interferon beta-1a in relapsing/remitting multiple sclerosis. PRISMS (Prevention of Relapses and Disability by Interferon beta-1a Subcutaneously in Multiple Sclerosis) Study Group. Lancet 1998; 352:1498-1504. 